\title{
Dermoscopic-Histopathological Correlation of Eccrine Poroma: An Observational Study
}

\author{
Marco A. Chessa ${ }^{1}$, Annalisa Patrizi ${ }^{1}$, Carlotta Baraldi ${ }^{1}$, Pier Alessandro Fanti ${ }^{1}$, \\ Alessia Barisani ${ }^{1}$, Sabina Vaccari ${ }^{1}$
}

1 Dermatology, Department of Experimental, Diagnostic and Specialty Medicine, University of Bologna, Italy

Key words: eccrine, poroma, dermoscopy, histopathology, diagnosis

Citation: Chessa MA, Patrizi A, Baraldi C, Fanti PA, Barisani A, Vaccari S. Dermoscopic-histopathological correlation of eccrine poroma: an observational study. Dermatol Pract Concept. 2019;9(4):283-291. DOI: https://doi.org/10.5826/dpc.0904a07

Accepted: July 15, 2019; Published: October 31, 2019

Copyright: $@ 2019$ Chessa et al. This is an open-access article distributed under the terms of the Creative Commons Attribution License, which permits unrestricted use, distribution, and reproduction in any medium, provided the original author and source are credited.

Funding: None.

Competing interests: The authors have no conflicts of interest to disclose.

Authorship: All authors have contributed significantly to this publication.

Corresponding author: Annalisa Patrizi, MD, Via Massarenti 1, 40138 Bologna, Italy. Email: alessiabarisani@gmail.com

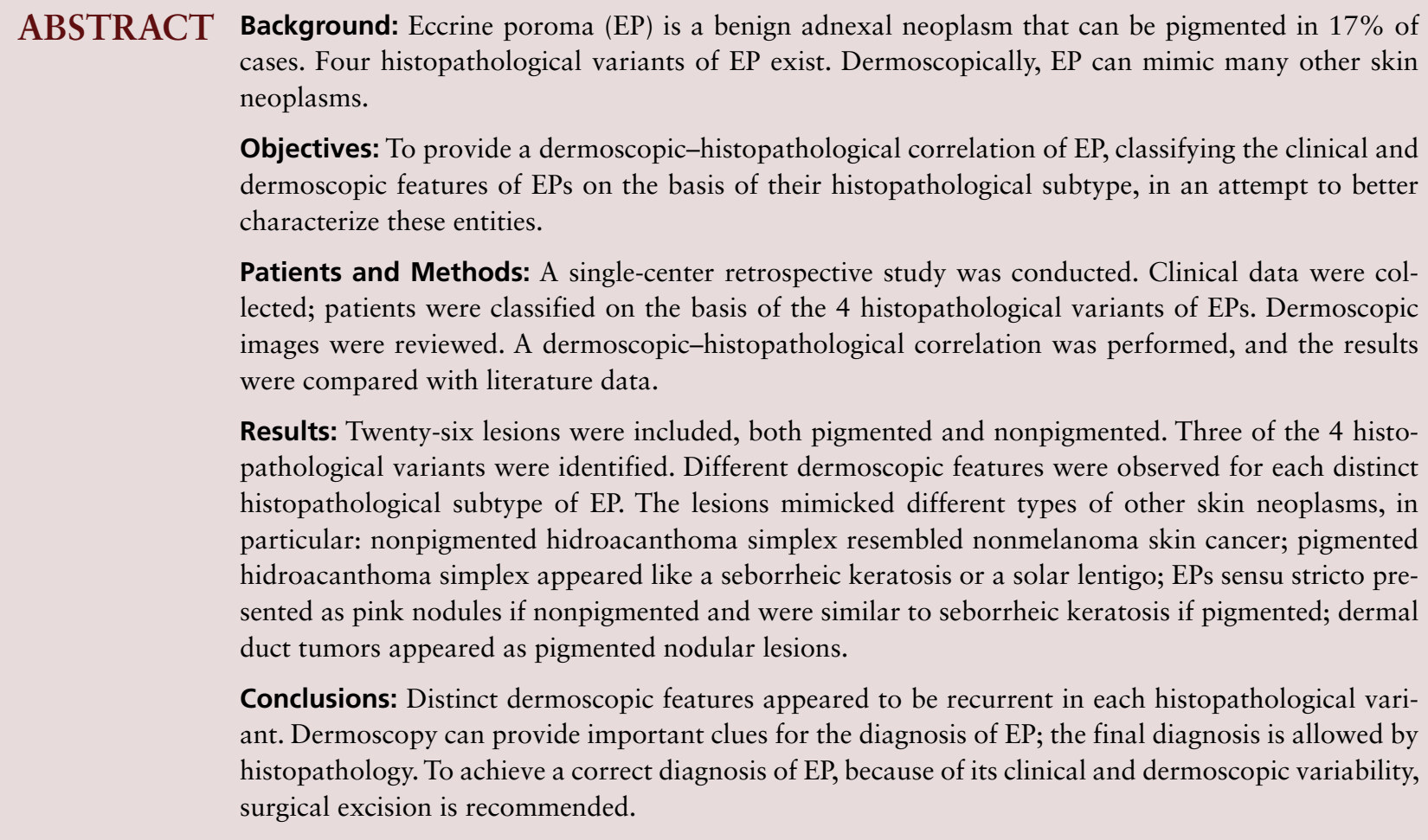




\section{Introduction}

Eccrine poroma (EP) is a benign adnexal neoplasm that originates from the segment of the intradermal and intraepidermal eccrine duct. EP usually appears as an asymptomatic single lesion and could be pigmented in $17 \%$ of cases [1]. Recently Lallas et al presented 8 characteristic dermoscopic patterns of EP, each one resembling a common skin tumor [2]. Because of its clinical and dermoscopic variability, EP is usually difficult to recognize and histopathology has a pivotal role in achieving the differential diagnosis. Four histopathological variants depending on the localization of poroid cells have been reported: hidroacanthoma simplex (HS), EP sensu stricto, dermal duct tumor (DDT), poroid hidradenoma (PH). In addition, EPs characterized by multiple histopathological variants, in the context of the same lesion, could be detected [3]. In the present study, we tried to provide a dermoscopic-histopathological correlation of EPs. The clinical and dermoscopic features of EPs were classified according to the histopathological subtype, in an attempt to assist the clinician who suspects this diagnosis.

\section{Methods}

Medical records and photographs were collected in a single-center retrospective study on EPs histopathologically detected in our Dermopathology Laboratory, Policlinico Sant'Orsola-Malpighi, University of Bologna, between January 2012 and December 2017. We recorded patients' sex, age at diagnosis of EPs, and morphology and distribution of the lesions. The cases were classified on the basis of the 4 possible histopathological variants of EPs, and each form was classified as pigmented or nonpigmented depending on the presence or absence of melanin within the lesion.

Dermoscopic images were achieved using a digital video camera system (Medicam 800; FotoFinder Systems GmbH, Bad Birnbach, Germany; original magnifications $\times 20-\times 40)$. Each dermoscopic image was then reviewed by 4 dermatologists. Concordance between dermatologists was considered when 3 out of 4 agreed on the dermoscopic structure.

A dermoscopic-histopathological correlation was performed for each lesion. On dermoscopy, the vascular patterns were described with the terms reported in the literature from inception to December 2017: glomerular or coiled vessels, linear irregular vessels, hairpin vessels, milky red areas, milky red globules, dotted vessels, helical vessels or corkscrew vessels, leaf-like vessels, flower-like vessels or cherry-blossom vessels or branched vessels with rounded endings, serpentine and chalice-form vessels [4-7]. The lesions with unique vessel morphology were considered monomorphous; those presenting any combination of $\geq 2$ vessel types were considered polymorphous. Finally, our results were compared with data from the literature.

The study was approved by the local ethics committee. All patients gave their informed consent to the study.

Descriptive statistics were performed to analyze the clinical and demographic data. Statistical analysis was performed using Stata/SE12.0 Statistical Software (STATA, College Station, TX).

\section{Results}

Twenty-six patients (14 men and 12 women) were included in this study; the mean age at diagnosis was 48.8 years (range 30-42 years). The lesions had been present for at least 1 year (range 1-20 years), without any significant changes in their clinical features over time. All lesions were asymptomatic and were detected incidentally during a routine dermatological visit.

In our case series, 26 histopathologically diagnosed EPs were included; 10 EPs were nonpigmented and 16 were pigmented. Clinically, all lesions were dome-shaped with well-defined borders and presented as a nodule in 11 cases and as a plaque in 15 cases. The main diameter of the EPs ranged from $6 \mathrm{~mm}$ to $25 \mathrm{~mm}$ (mean $11 \mathrm{~mm}$ ). Different body areas were affected, in particular the trunk ( 9 cases), the face or scalp ( 7 cases), the feet or lower limbs (6 cases), and the abdomen (4 cases). All tumors were excised since a definitive diagnosis was not possible on the basis of clinical and dermoscopic features. A clinical/dermoscopic initial suspicion of EP was reported in only 5 cases.

On histopathology, all lesions were characterized by the presence of small, dark, monomorphous, cuboidal poroid cells; pale and larger cuticular cells; and intracytoplasmatic or intercellular vacuolization. Three of the 4 variants of EPs were identified in our case series, considering the distribution of poroid cells through the epidermidis and the dermis (Figure 1). The fourth variant (PH), as well as EPs characterized by multiple histopathological variants in the context of the same lesion, was not detected in our case series.

A dermoscopic-histopathological correlation was made in all cases. The 4 cases diagnosed as nonpigmented hidroacanthoma simplex (NPHS) clinically resembled nonmelanoma skin cancers (NMSCs), such as Bowen disease (BD) (3 cases) or squamous cell carcinoma (SCC) (1 case); on dermoscopy, polymorphous vessels were detected in all cases, consisting of glomerular, linear irregular, flower-like vessels and corkscrew vessels. Milia-like cysts were also present (Figure 2, A-C).

The 5 cases of pigmented hidroacanthoma simplex (PHS) appeared as a flat seborrheic keratosis (SK) or a solar lentigo (SL), respectively. On dermoscopy, network-like structures 


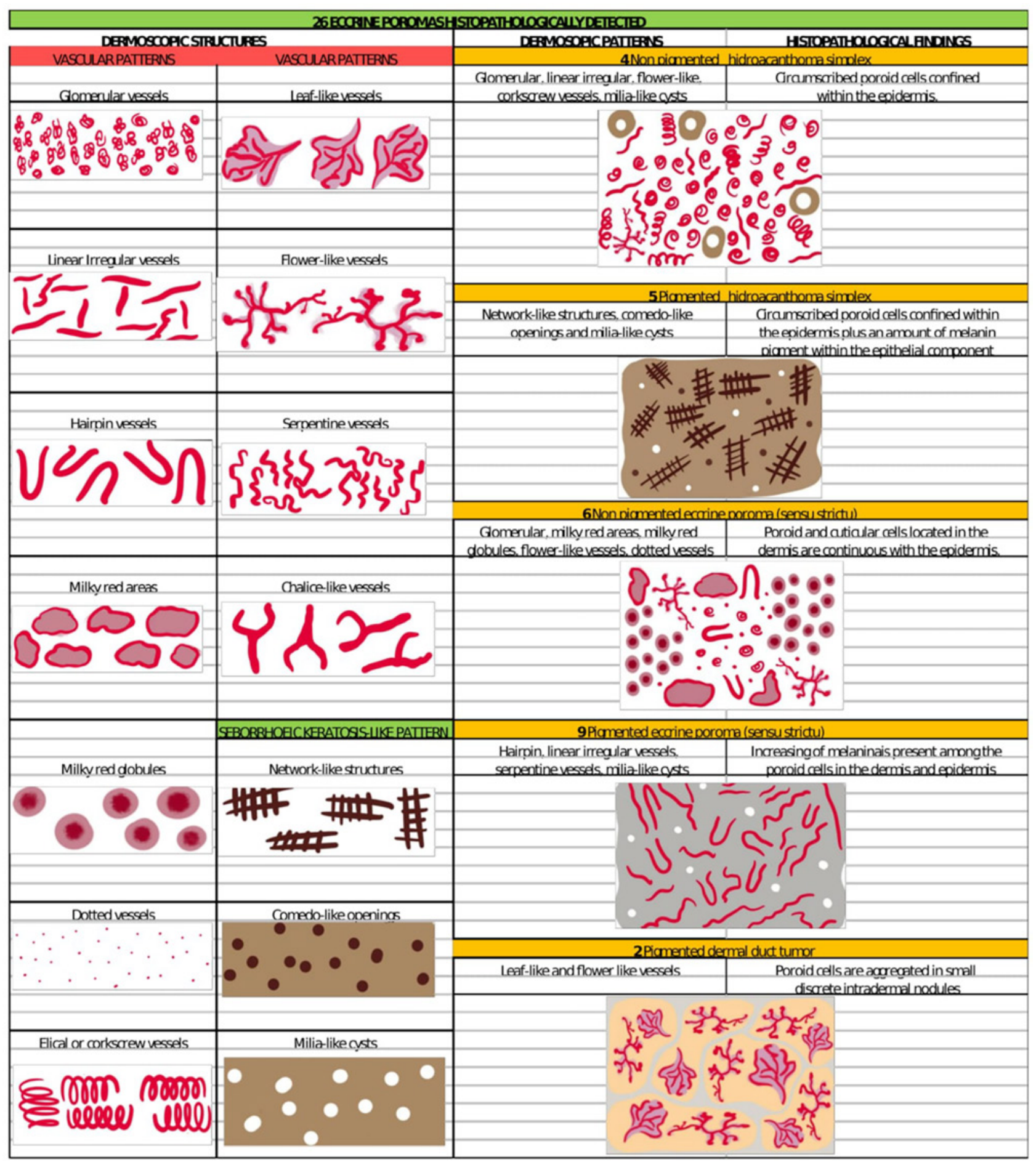

Figure 1. Dermoscopic and histopathological findings of the 26 eccrine poromas included in the study. [Copyright: ${ }_{0} 2019$ Chessa et al.]

around follicles, comedo-like openings, and milia-like cysts were detected. In these cases a vascular pattern was not present (Figure 3, A-C).

All 6 cases of nonpigmented eccrine poroma (NPEP) sensu stricto clinically appeared as pink nodules. On dermoscopy, a polymorphous vascular pattern (including at least 2 types of vascular structures) was detected in all cases: glomerular vessels, milky red globules, milky red areas, flower-like vessels, and dotted vessels (Figure 4, A-C). In 5 out of 6 cases, a diagnosis of EP was proposed by clinicians considering these dermoscopic features, whereas in the sixth case, an amelanotic melanoma was suspected.

The 9 cases of pigmented eccrine poroma (PEP) sensu stricto resembled an SK based on the clinical and dermoscopic features. Comedo-like openings, milia-like cysts, and a cerebriform pattern were detected. The lesions were characterized by hairpin vessels in the central portion and linear irregular and serpentine vessels peripherally (Figure 5, A-C).

The 2 cases of pigmented dermal duct tumor (PDDT) appeared as pigmented nodules of the scalp and the lower 


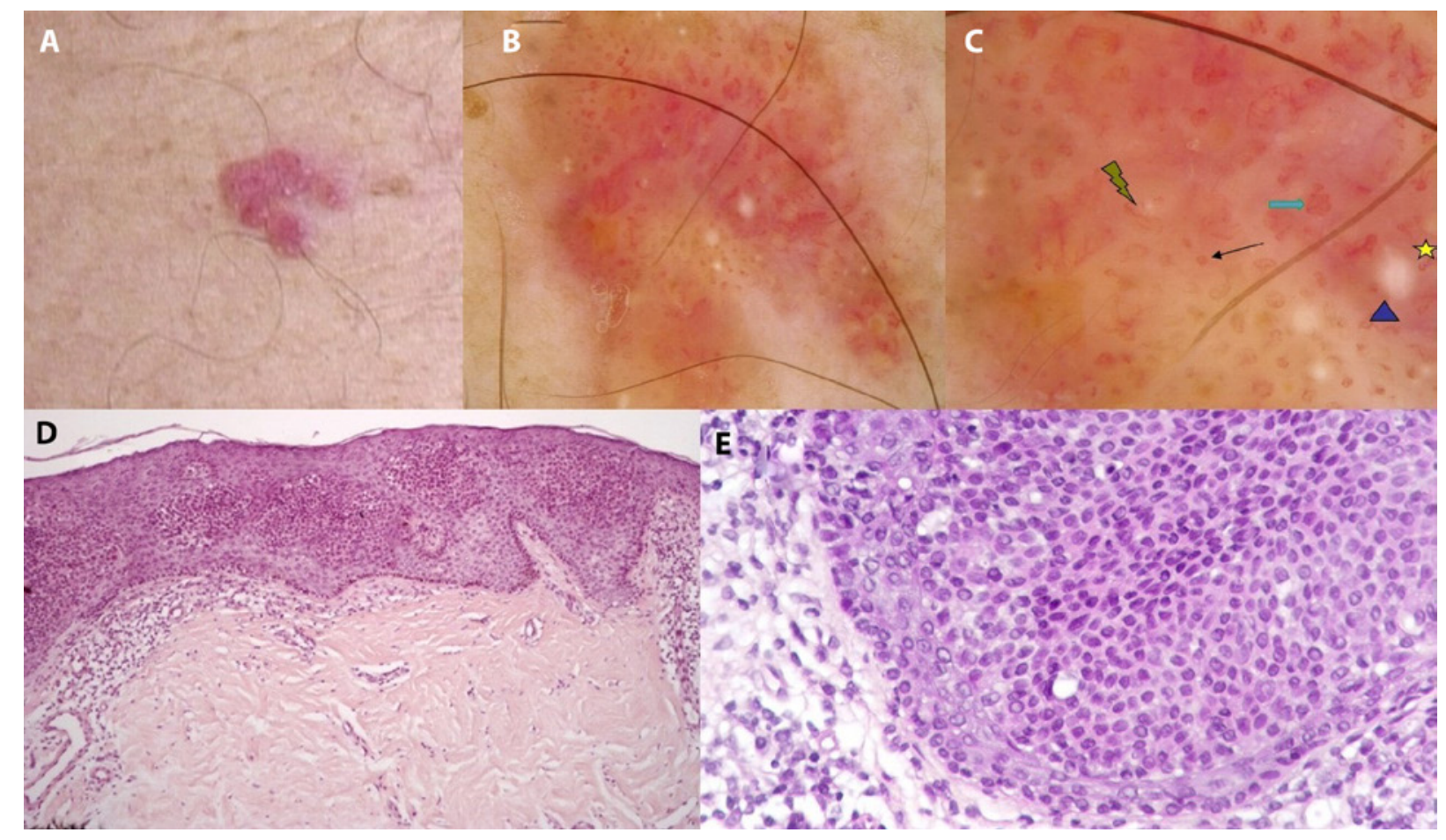

Figure 2. (A) Nonpigmented hidroacanthoma simplex (NPHS) appearing as a pink plaque of the hip. (B,C) On dermoscopy, glomerular (black arrow), linear irregular (green bolt), flower-like (green arrow), and corkscrew vessels (yellow star) and milia-like cysts (dark blue triangle) were observed (original magnifications $\times 20$ and $\times 40$, respectively). (D) Histopathological features of NPHS were detected, consisting of well-circumscribed poroid cells arranged in an ovoid aggregation confined within the epidermis (H\&E staining, original magnification $\times 4$ ). (E) At higher magnification, sharply delineated aggregations of poroid cells that spare the basal layer of the epidermis and dark-staining pyknotic nuclei can be seen better (H\&E staining, original magnification $\times 40$ ). [Copyright: @2019 Chessa et al.]

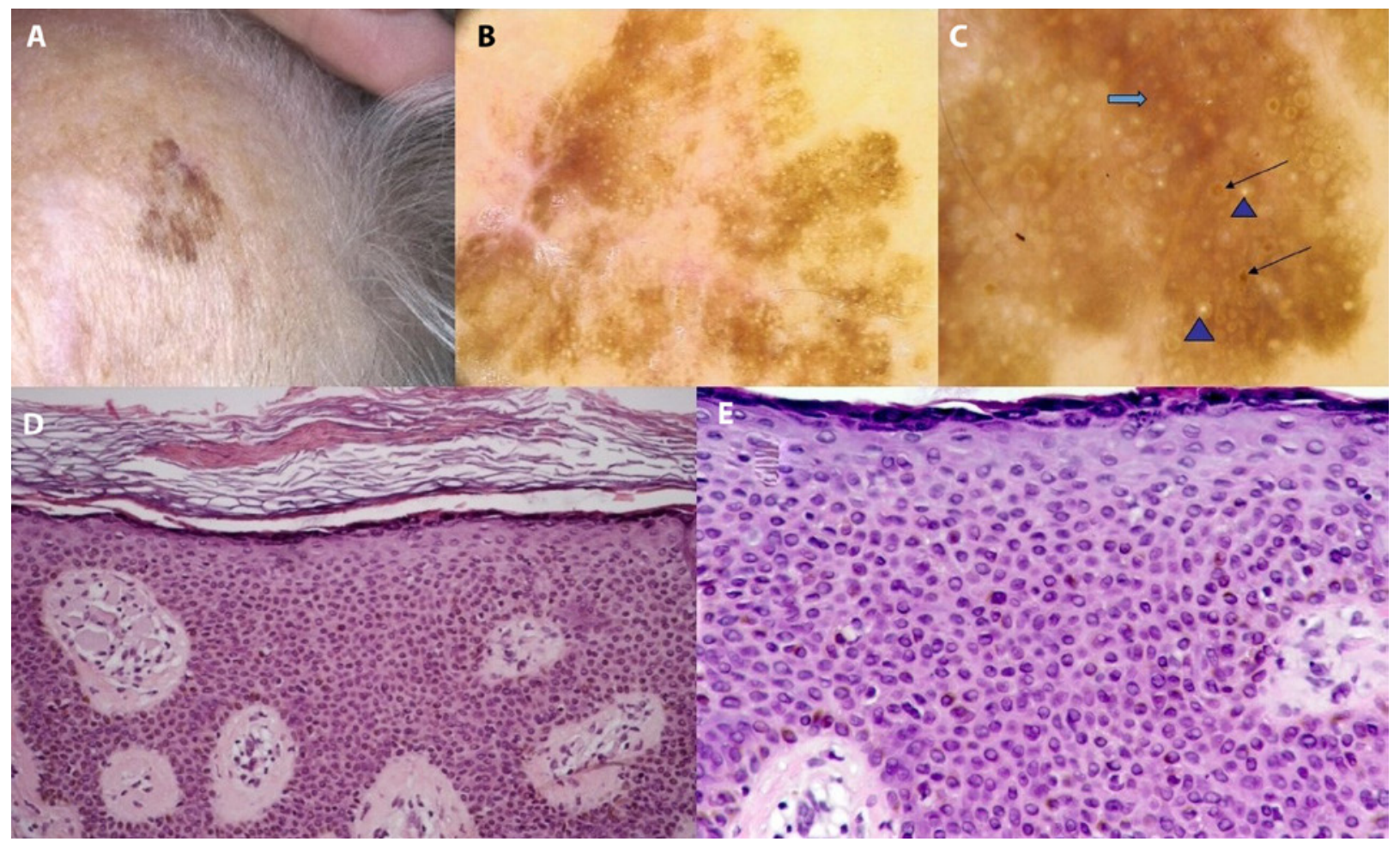

Figure 3. (A) Pigmented hidroacanthoma simplex appearing as a dark brown plaque located on the forehead. (B,C) On dermoscopy, a brown pseudonetwork with fingerprint-like structures around follicles (light blue arrow), milia-like cysts (dark blue triangle), and comedo-like openings (black arrow) were seen, mimicking a seborrheic keratosis (original magnifications $\times 20$ and $\times 40$, respectively). (D) On histopathology, sharply delineated aggregation of poroid, cuticular cells and tubular structures, and an increase of melanin pigment among the epidermis, were observed (H\&E staining, original magnification $\times 10$ ). (E) At higher magnification, small, dark, monomorphous, neoplastic poroid cells constitute most of the sharply circumscribed aggregations within the epidermis; these findings are diagnostic of hidroacanthoma simplex (H\&E staining, original magnification $\times 40)$. [Copyright: (O2019 Chessa et al.] 


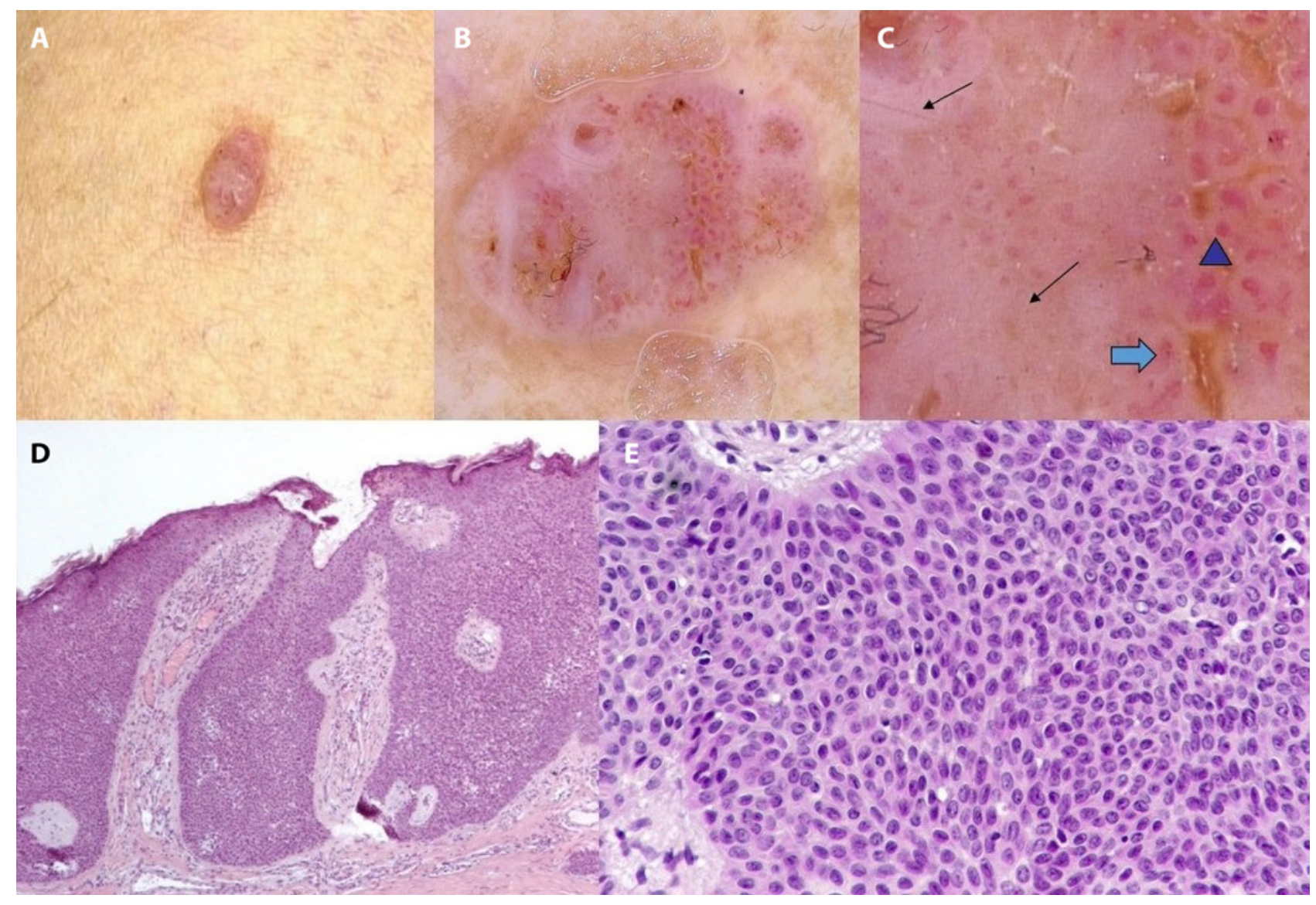

Figure 4. (A) Clinical presentation of a nonpigmented eccrine poroma sensu stricto as a pink nodule located on the foot. (B,C) Dermoscopy shows milky red areas (light blue arrow), milky red globules (dark blue triangle), and dotted vessels (black arrow) (original magnifications $\times 20$ and $\times 40$, respectively). (D,E) Histopathologically, the neoplasm consists of poroid and cuticular cells and tubular structures which are continuous with the epidermis (H\&E staining, original magnifications $\times 10$ and $\times 40$, respectively). [Copyright: @2019 Chessa et al.]

limb. In the first case, gray-yellow pigmented lobules separated by grayish septa with leaf-like and flower-like vessels were detected within the peripheral lobules (Figure 6, A-C). The second PDDT showed yellow pigmented lobules separated by grayish septa and monomorphous, flower-like vessels (Figure 7, A-C).

Polymorphous vascular structures were found in 20 out of 26 EPs. In 4 cases, a vascular pattern was not identifiable. The most frequently detected vascular patterns were linear irregular vessels in 11 cases and glomerular vessels in 10 cases. In 6 cases milky red globules and in 4 cases milky red areas were observed (Figure 1).

\section{Discussion}

In the literature, EP is often reported as a single entity, without any specification of the histopathological subtype [1,2]. The great clinical and dermoscopic variability within the category of EPs is related to the distribution of the cells in the epidermis and the dermis.
HS is histopathologically characterized by nests of poroid cells confined to the epidermis, whereas clinically the lesions often appear as plaques [8].

NPHS often resembles an NMSC, such as BD and SCC [8]. In $\mathrm{BD}$, scales are usually observed in the entire lesion, and glomerular vessels are a hallmark in $90 \%$ of cases [9]. In SCC, atypical keratinization is typically prominent in the central part of the lesion, whereas in the periphery, polymorphous vessels, including linear irregular or serpentine vessels, could be detected on a white background [10,11]. However, the dermoscopic criteria useful to distinguish HS from BD [12] were not confirmed in our case series: in fact, glomerular vessels were detected also in NPHS (Figure 2, B and C); moreover, polymorphous vascular structures, such as linear irregular and glomerular vessels, were detected in NPHS, similarly to SCC (Figure 2, B and C). Instead, the presence of milia-like cysts in NPHS (Figure 2, B and C) might be a useful criterion for the differential diagnosis with BD and SCC.

PHS could resemble a flat SK or an SL, as in our case series. However, SL is typically characterized by a structureless homogenous pigmentation and numerous openings of 


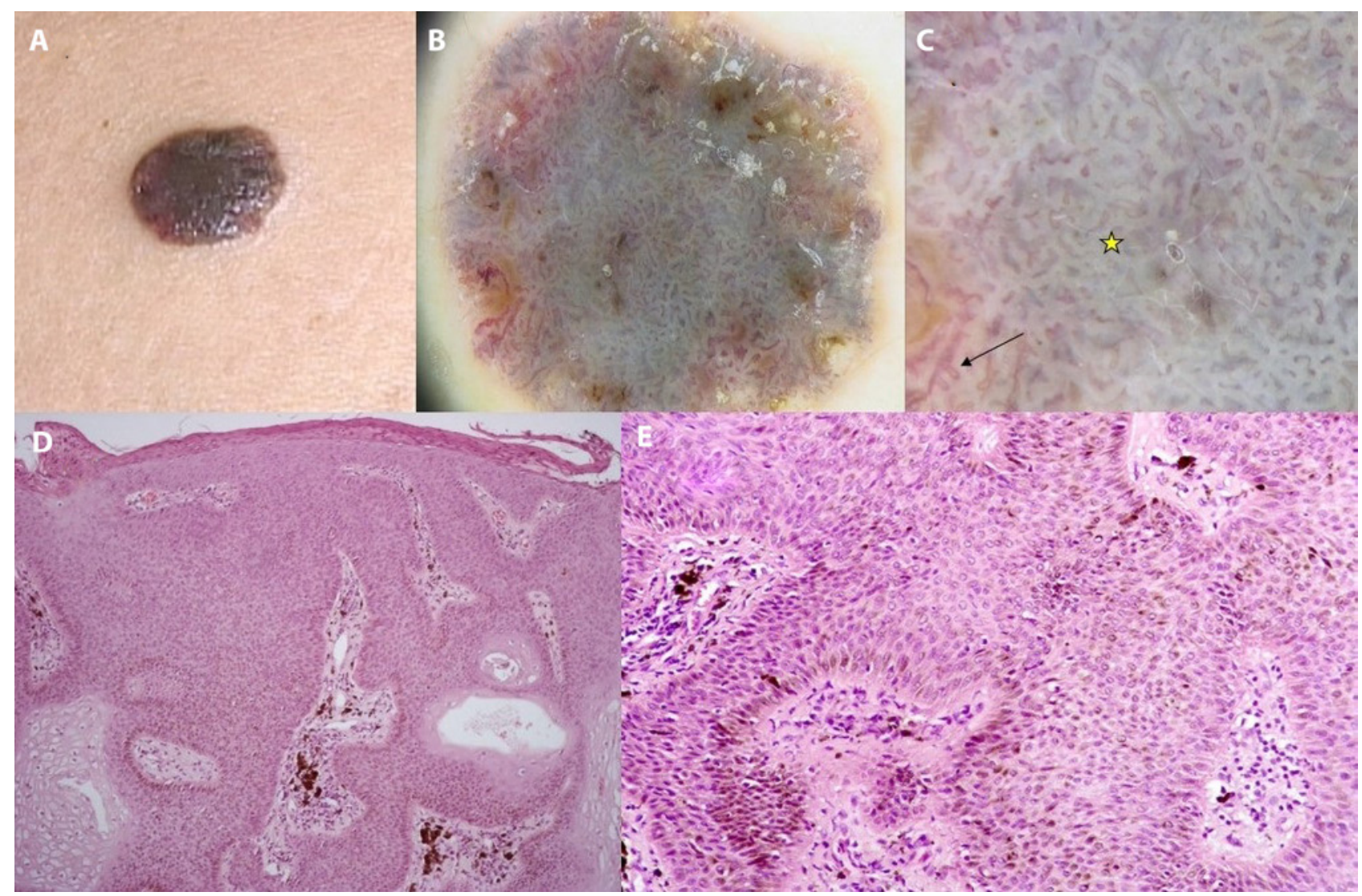

Figure 5. (A) A pigmented eccrine poroma (PEP) presenting as a well-defined, pigmented seborrheic keratosis-like lesion of the abdomen. (B,C) Dermoscopy shows a gray-to-brown background with a uniform distribution of the vascular structures, consisting mainly of hairpin vessels (yellow star) in the central part and linear irregular (yellow arrow) and serpentine vessels (black arrow) at the periphery of the PEP (original magnifications $\times 20$ and $\times 40$, respectively). (D) Histopathologically, an increase of melanin pigment is present among the PEP (H\&E staining, original magnification $\times 10$ ). (E) At higher magnification, the 2 cell types that constitute poromas are seen: cells with small dark-staining nuclei and scant cytoplasm are poroid cells, whereas cells with larger, paler nuclei and abundant cytoplasm are cuticular cells (H\&E staining, original magnification $\times 20$ ). [Copyright: @2019 Chessa et al.]

hair follicles [13]. In contrast, PHS appeared to be characterized by an irregular pseudonetwork, some milia-like cysts, and comedo-like openings that could help rule out an SL (Figure 3C). Like in flat SKs, also our case of PHS (Figure 3C) showed fingerprint-like structures around the hair follicles and comedo-like openings; however, the patient did not present any other similar lesions on the skin; therefore, an incisional biopsy was performed. In the literature, these dermoscopic and histopathological findings were reported when incisional biopsies were performed in the thinner part of the lesions $[14,15]$.

The most common clinical presentation of NPEP is a firm nodule, from pink to red in color. These lesions are often characterized by polymorphous vessels [16]. On dermoscopy, many terms were used to describe the vascular patterns of NPEPs: structureless pink-white areas, milky red areas or red globular structures, lacuna-like areas, glomerular, chalice-form, cherry-blossom, flower-like, and leaf-like vessels [16-21]. The differential diagnosis includes several malignant lesions, such as BD, SCC, and amelanotic melanoma. Anam- nestic data may be useful for the differential diagnosis, as NPEPs are generally reported as having been present for a long time, without any substantial variations.

PEPs represent a small percentage of EPs. These lesions may resemble an SK on dermoscopy, because comedo-like openings, milia-like cysts, and hairpin vessels are usually detected [1,22]. However, clinical and dermoscopic features may show some alarming features, such as blue-gray areas and polymorphous vessels [23], resembling a pigmented basal cell carcinoma (BCC) or a melanoma.

DDT usually appears as papulonodules. Dark brown roundish structures or dark bluish structureless areas may be observed [24]. A lobular overall appearance, histopathologically consisting of interlacing white cords, which separate gray-bluish structureless islands, has been reported, as well in our study, with or without vascular structures inside the islands [24,25]. We detected leaf-like and flower-like vessels in the peripheral lobules (Figures 6C and 7C). Histopathology is needed to rule out a nodular melanoma or a pigmented BCC. 


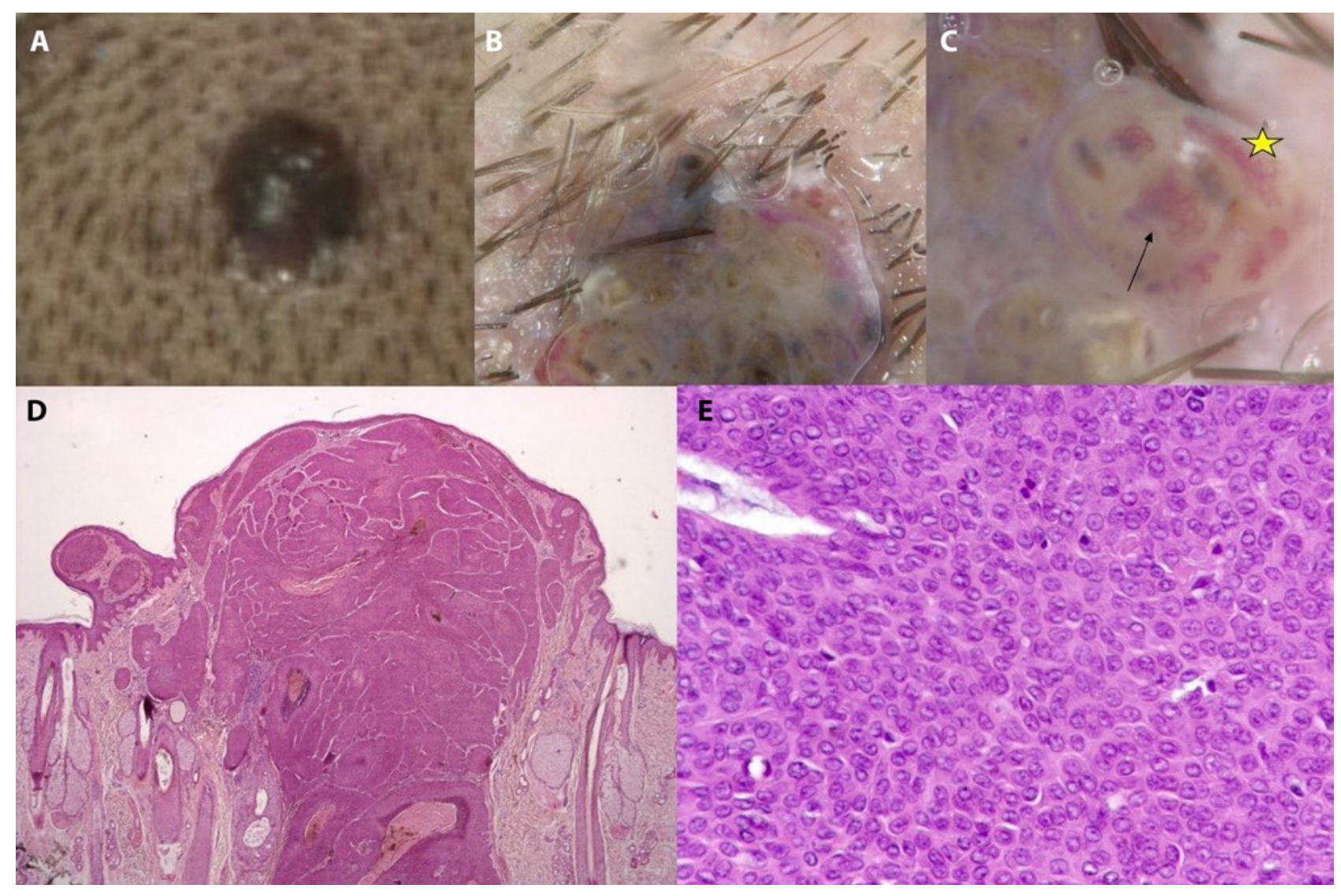

Figure 6. (A) A pigmented dermal duct tumor appearing as a pigmented nodule of the scalp. (B,C) Dermoscopy reveals light brown, pigmented lobules separated by grayish septa, with some leaf-like (yellow star) and flower-like vessels (black arrow) in some peripheral lobules (original magnifications $\times 20$ and $\times 40$, respectively). (D) Histopathological features consist of poroid cells and cuticular cells aggregated in small, discrete intradermal nodules (H\&E staining, original magnification $\times 4$ ). (E) At higher magnification, vacuoles are visible within cuticular cells and represent a stage en route to formation of ducts (H\&E staining, original magnification $\times 40$ ). [Copyright: $\odot 2019$ Chessa et al.]

PH may appear as a pigmented or a nonpigmented nodule, usually with a nonspecific, polymorphic vascular pattern; the differential diagnosis with amelanotic melanoma and ulcerated SCC requires surgical excision [26].

EPs characterized by multiple histopathological variants in the context of the same lesion usually show multiple colors, with thick vessels and large blue-gray ovoid nests, resembling a pigmented BCC $[1,27]$. These EPs were scarcely reported in the literature, and their surgical excision is always suggested.

Polymorphous vessels ( $77 \%$ of cases in our study) are frequently associated with EPs; this data was confirmed by Marchetti et al in a recent cross-sectional, observational study on 113 EPs [28]. According to the authors, milky red globules could be considered a dermoscopic feature specifically associated with EPs [28].

EP has been named "the great dermoscopic imitator" owing to its clinical and, above all, dermoscopic variability. In the present work, we described their dermoscopic features on the basis of the histopathological subtype.

Limitations of this study are represented by the rarity of EPs, which are often underestimated and make it difficult to achieve a great number of cases and to find significant relationships from the data.

Despite these limitations, we were able to find 3 of the main histopathological variants and found that several dermoscopic features were recurrent in each histopathological variant (Figure 1). To the best of our knowledge, no previous studies have investigated the dermoscopic-histopathological correlations of EPs. Further case series, and larger case numbers, may strengthen these associations.

\section{Conclusions}

In the presence of a well-defined nodule or plaque, either pigmented or nonpigmented, with a low tendency to change over several years, the clinical suspicion of EP should be hypothesized. Our data show that dermoscopy may provide important clues for this diagnosis; in particular, it can show the recurrence of distinct patterns in the different histopathological subtypes. NPHS can often resemble an NMSC; PHS may mimic an SK or an SL; EP sensu stricto may present as pink nodules, or like an SK if pigmented; 


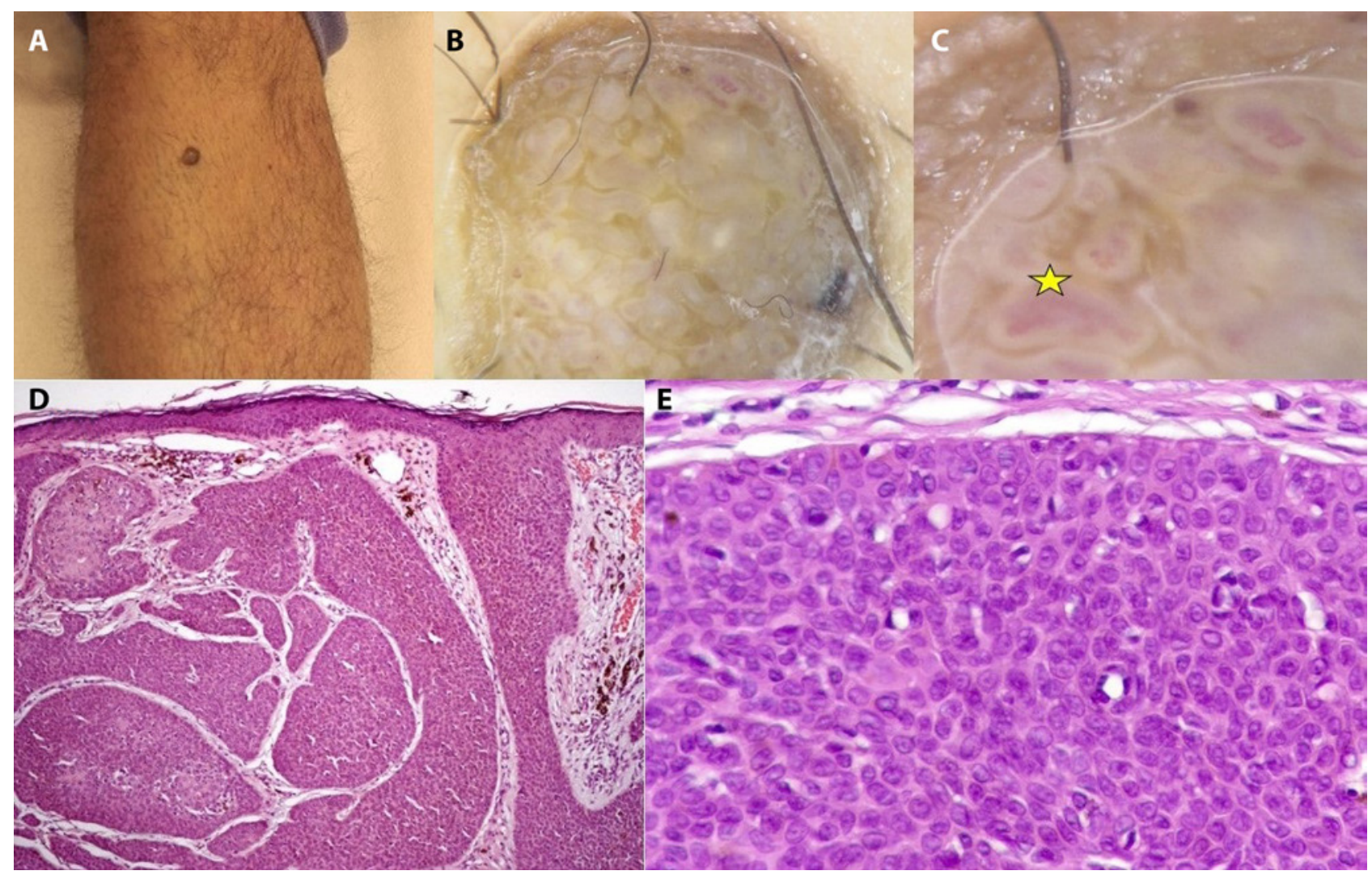

Figure 7. (A) A pigmented dermal duct tumor presenting as a pigmented nodule of the lower limb. (B,C) Dermoscopy shows yellow lobules separated by grayish septa, with flower-like vessels (yellow star) in some peripheral lobules (original magnifications $\times 20$ and $\times 40$, respectively). (D) Histopathology shows poroid and cuticular cells aggregated in intradermal nodules with an increase of melanin pigment (H\&E staining, original magnification $\times 10$ ). (E) At higher magnification, the sequence of changes that leads to formation of ductal structures is visible; the earliest changes consist of tiny vacuoles within the cytoplasm of cuticular cells (H\&E staining, original magnification $\times 40$ ). [Copyright: (C2019 Chessa et al.]

DDT is often a pigmented nodule. However, a definitive diagnosis based on clinical and dermoscopic findings is generally challenging. Because these benign neoplasms can often resemble other skin neoplasms, including BCC, SCC, and malignant melanoma, and because their transformation into a porocarcinoma should be kept in mind [29], surgical excision is always recommended.

\section{References}

1. Minagawa A, Koga H. Dermoscopy of pigmented poromas. Dermatology. 2010;221(1):78-83.

2. Lallas A, Chellini PR, Guimarães MG, et al. Eccrine poroma: the great dermoscopic imitator. J Eur Acad Dermatol Venereol. 2016;30(10):e61-e63.

3. Battistella M, Langbein L, Peltre B, Cribier B. From hidroacanthoma simplex to poroid hidradenoma: clinicopathologic and immunohistochemic study of poroid neoplasms and reappraisal of their histogenesis. Am J Dermatopathol. 2010;32(5):459-468.

4. Martín JM, Bella-Navarro R, Jordá E. Vascular patterns in dermoscopy. Actas Dermosifiliogr. 2012;103(5):357-375.
5. Ayhan E, Ucmak D, Akkurt Z. Vascular structures in dermoscopy. An Bras Dermatol. 2015;90(4):545-553.

6. Togawa Y. Review of vasculature visualized on dermoscopy. $J$ Dermatol. 2017;44(5):525-532.

7. Lallas A, Giacomel J, Argenziano G, et al. Dermoscopy in general dermatology: practical tips for the clinician. $\mathrm{Br} J$ Dermatol. 2014;170(3):514-526.

8. Furlan KC, Kakizaki P, Chartuni JCN, Sittart JA, Valente NYS. Hidroacanthoma simplex: dermoscopy and cryosurgery treatment. An Bras Dermatol. 2017;92(2):253-255.

9. Zalaudek I, Argenziano G, Leinweber B, et al. Dermoscopy of Bowen's disease. Br J Dermatol. 2004;150(6):1112-1116.

10. Zalaudek I, Giacomel J, Schmid K, et al. Dermatoscopy of facial actinic keratosis, intraepidermal carcinoma, and invasive squamous cell carcinoma: a progression model. J Am Acad Dermatol. 2012;66(4):589-597.

11. Russo T, Piccolo V, Lallas A, et al. Dermoscopy of malignant skin tumours: what's new? Dermatology. 2017;233(1):64-73.

12. Shiiya C, Hata H, Inamura Y, et al. Dermoscopic features of hidroacanthoma simplex: usefulness in distinguishing it from Bowen's disease and seborrheic keratosis. J Dermatol. 2015;42(10):10021005. 
13. Goncharova Y, Attia EA, Souid K, Vasilenko IV. Dermoscopic features of facial pigmented skin lesions. ISRN Dermatol. 2013;2013:546813. Epub 2013 Feb 3.

14. Dong H, Zhang H, Liu N, Soyer HP. Dermoscopy of a pigmented apocrine porocarcinoma arising from a pigmented hidroacanthoma simplex. Australas J Dermatol. 2018;59(2):e151-e152.

15. Sato Y, Fujimura T, Tamabuchi E, Haga T, Aiba S. Dermoscopy findings of hidroacanthoma simplex. Case Rep Dermatol. 2014;6(2):154-158.

16. Espinosa AE, Ortega BC, Venegas RQ, Ramírez RG. Dermoscopy of non-pigmented eccrine poromas: study of Mexican cases. Dermatol Pract Concept. 2013;3(1):25-28.

17. Nicolino R, Zalaudek I, Ferrara G, et al. Dermoscopy of eccrine poroma. Dermatology. 2007;215(2):160-163.

18. Ferrari A, Buccini P, Silipo V, et al. Eccrine poroma: a clinical-dermoscopic study of seven cases. Acta Derm Venereol. 2009;89(2):160-164.

19. Sgouros D, Piana S, Argenziano G, et al. Clinical, dermoscopic and histopathological features of eccrine poroid neoplasms. Dermatology. 2013;227(2):175-179.

20. Aydingoz IE. New dermoscopic vascular patterns in a case of eccrine poroma. J Eur Acad Dermatol Venereol. 2009;23(6):725726.

21. Argenziano G, Zalaudek I, Corona R, et al. Vascular structures in skin tumors: a dermoscopy study. Arch Dermatol. 2004;140(12):1485-1489.
22. Almeida FC, Cavalcanti SM, Medeiros AC, Teixeira MA. Pigmented eccrine poroma: report of an atypical case with the use of dermoscopy. An Bras Dermatol. 2013;88(5):803-806.

23. Bombonato C, Piana S, Moscarella E, Lallas A, Argenziano G, Longo C. Pigmented eccrine poroma: dermoscopic and confocal features. Dermatol Pract Concept. 2016;6(3):59-62.

24. Oiso N, Matsuda H, Kawada A. Biopsy-proven pigmented poroma with no vascular structure in dermoscopy. Int J Dermatol. 2014;53(6):e334-e335.

25. Shalom A, Schein O, Landi C, Marghoob A, Carlos B, Scope A. Dermoscopic findings in biopsy-proven poromas. Dermatol Surg. 2012;38(7 Pt 1):1091-1096.

26. Robles-Mendez JC, Martınez-Cabriales SA, Villarreal-Martinez A, et al. Nodular hidradenoma: dermoscopic presentation. J Am Acad Dermatol. 2017;76(2S1):S46-S48.

27. Ichiyama S, Hoashi T, Funasaka Y, et al. Pigmented poroma on the temporal region dermoscopically mimicking basal cell carcinoma: a report of two cases. J Dermatol. 2018;45(4):e94-e95.

28. Marchetti MA, Marino ML, Virmani P, et al. Dermoscopic features and patterns of poromas: a multicentre observational case-control study conducted by the International Dermatoscopy Society. J Eur Acad Dermatol Venereol. 2018;32(8):1263-1271.

29. Shaw M, McKee PH, Lowe D, Black MM. Malignant eccrine poroma: a study of twenty-seven cases. Br J Dermatol. 1982;107(6):675-680. 